\title{
Introduction to biosensors
}

\author{
Nikhil Bhalla, Pawan Jolly, Nello Formisano and Pedro Estrela
}

Department of Electronic and Electrical Engineering, University of Bath, Bath BA2 7AY, U.K.

Correspondence: Pedro Estrela (p.estrela@bath.ac.uk)

Biosensors are nowadays ubiquitous in biomedical diagnosis as well as a wide range of other areas such as point-of-care monitoring of treatment and disease progression, environmental monitoring, food control, drug discovery, forensics and biomedical research. A wide range of techniques can be used for the development of biosensors. Their coupling with high-affinity biomolecules allows the sensitive and selective detection of a range of analytes. We give a general introduction to biosensors and biosensing technologies, including a brief historical overview, introducing key developments in the field and illustrating the breadth of biomolecular sensing strategies and the expansion of nanotechnological approaches that are now available.

\section{Introduction}

A biosensor is a device that measures biological or chemical reactions by generating signals proportional to the concentration of an analyte in the reaction. Biosensors are employed in applications such as disease monitoring, drug discovery, and detection of pollutants, disease-causing micro-organisms and markers that are indicators of a disease in bodily fluids (blood, urine, saliva, sweat). A typical biosensor is represented in Figure 1; it consists of the following components.

- Analyte: A substance of interest that needs detection. For instance, glucose is an 'analyte' in a biosensor designed to detect glucose.

- Bioreceptor: A molecule that specifically recognises the analyte is known as a bioreceptor. Enzymes, cells, aptamers, deoxyribonucleic acid (DNA) and antibodies are some examples of bioreceptors. The process of signal generation (in the form of light, heat, $\mathrm{pH}$, charge or mass change, etc.) upon interaction of the bioreceptor with the analyte is termed bio-recognition.

- Transducer: The transducer is an element that converts one form of energy into another. In a biosensor the role of the transducer is to convert the bio-recognition event into a measurable signal. This process of energy conversion is known as signalisation. Most transducers produce either optical or electrical signals that are usually proportional to the amount of analyte-bioreceptor interactions.

- Electronics: This is the part of a biosensor that processes the transduced signal and prepares it for display. It consists of complex electronic circuitry that performs signal conditioning such as amplification and conversion of signals from analogue into the digital form. The processed signals are then quantified by the display unit of the biosensor.

- Display: The display consists of a user interpretation system such as the liquid crystal display of a computer or a direct printer that generates numbers or curves understandable by the user. This part often consists of a combination of hardware and software that generates results of the biosensor in a

Version of Record published 30 June 2016 user-friendly manner. The output signal on the display can be numeric, graphic, tabular or an image, depending on the requirements of the end user. 


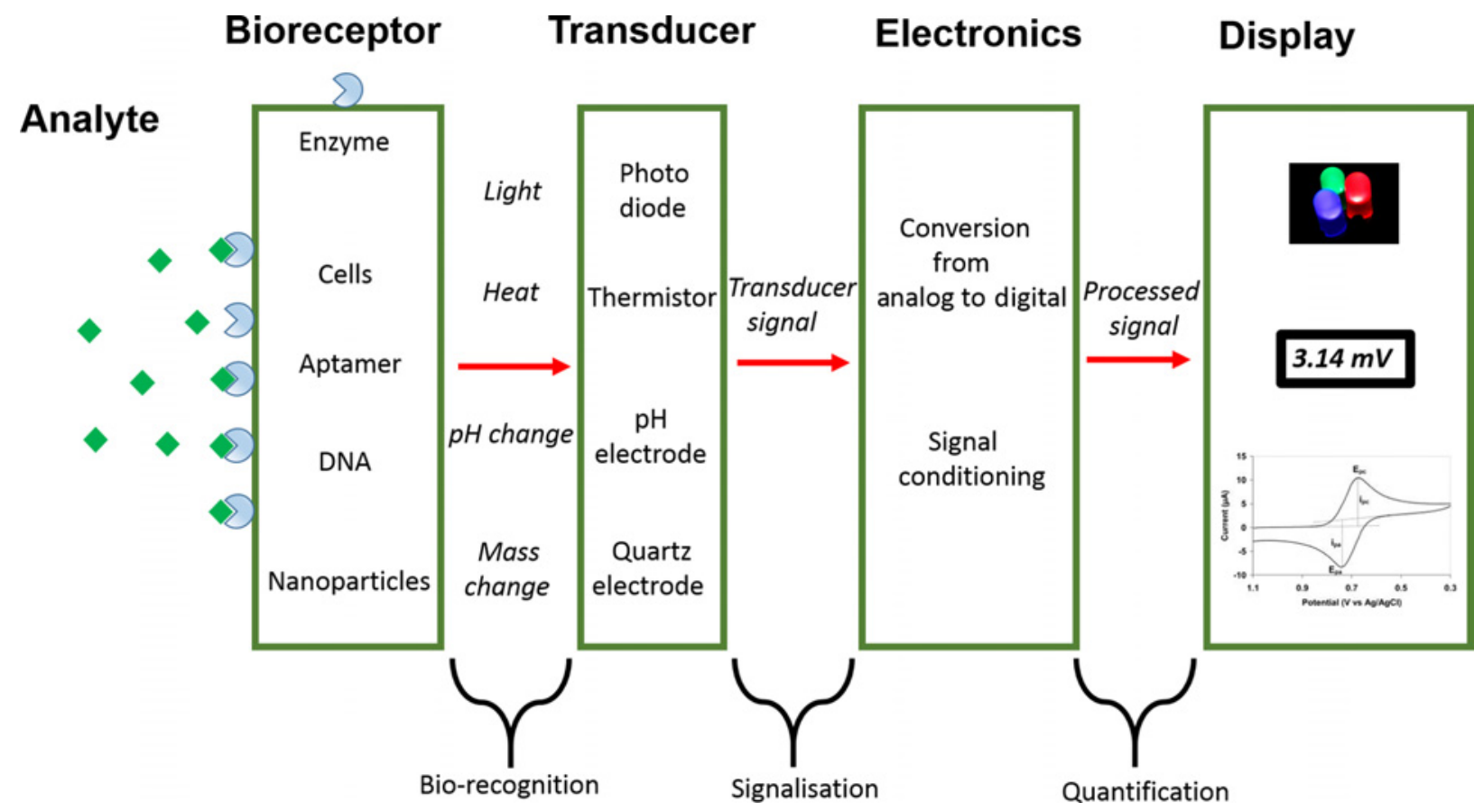

Figure 1. Schematic representation of a biosensor

\section{Historical background}

The history of biosensors dates back to as early as 1906 when M. Cremer [1] demonstrated that the concentration of an acid in a liquid is proportional to the electric potential that arises between parts of the fluid located on opposite sides of a glass membrane. However, it was only in 1909 that the concept of $\mathrm{pH}$ (hydrogen ion concentration) was introduced by Søren Peder Lauritz Sørensen and an electrode for $\mathrm{pH}$ measurements was realised in the year 1922 by W.S. Hughes [2]. Between 1909 and 1922, Griffin and Nelson [3,4] first demonstrated immobilisation of the enzyme invertase on aluminium hydroxide and charcoal. The first 'true' biosensor was developed by Leland C. Clark, Jr in 1956 for oxygen detection. He is known as the 'father of biosensors' and his invention of the oxygen electrode bears his name: 'Clark electrode' [5]. The demonstration of an amperometric enzyme electrode for the detection of glucose by Leland Clark in 1962 was followed by the discovery of the first potentiometric biosensor to detect urea in 1969 by Guilbault and Montalvo, Jr [6]. Eventually in 1975 the first commercial biosensor was developed by Yellow Spring Instruments (YSI). Table 1 shows the historical overview of biosensors in the period 1970-1992. Ever since the development of the i-STAT sensor, remarkable progress has been achieved in the field of biosensors. The field is now a multidisciplinary area of research that bridges the principles of basic sciences (physics, chemistry and biology) with fundamentals of micro/nano-technology, electronics and applicatory medicine. The database 'Web of Science' has indexed over 84000 reports on the topic of 'biosensors' from 2005 to 2015.

\section{Characteristics of a biosensor}

There are certain static and dynamic attributes that every biosensor possesses. The optimisation of these properties is reflected on the performance of the biosensor. 
Table 1. Important cornerstones in the development of biosensors during the period 1970-1992

\begin{tabular}{ll}
1970 & Discovery of ion-sensitive field-effect transistor (ISFET) by Bergveld [7] \\
1975 & Fibre-optic biosensor for carbon dioxide and oxygen detection by Lubbers and Opitz [8] \\
1975 & First commercial biosensor for glucose detection by YSI [9] \\
1975 & First microbe-based immunosensor by Suzuki et al. [10] \\
1982 & Fibre-optic biosensor for glucose detection by Schultz [11] \\
1983 & Surface plasmon resonance (SPR) immunosensor by Liedberg et al. [12] \\
1984 & First mediated amperometric biosensor: ferrocene used with glucose oxidase for glucose detection [13] \\
1990 & SPR-based biosensor by Pharmacia Biacore [8] \\
1992 & Handheld blood biosensor by i-STAT [8] \\
\hline
\end{tabular}

\section{Selectivity}

Selectivity is perhaps the most important feature of a biosensor. Selectivity is the ability of a bioreceptor to detect a specific analyte in a sample containing other admixtures and contaminants. The best example of selectivity is depicted by the interaction of an antigen with the antibody. Classically, antibodies act as bioreceptors and are immobilised on the surface of the transducer. A solution (usually a buffer containing salts) containing the antigen is then exposed to the transducer where antibodies interact only with the antigens. To construct a biosensor, selectivity is the main consideration when choosing bioreceptors.

\section{Reproducibility}

Reproducibility is the ability of the biosensor to generate identical responses for a duplicated experimental set-up. The reproducibility is characterised by the precision and accuracy of the transducer and electronics in a biosensor. Precision is the ability of the sensor to provide alike results every time a sample is measured and accuracy indicates the sensor's capacity to provide a mean value close to the true value when a sample is measured more than once. Reproducible signals provide high reliability and robustness to the inference made on the response of a biosensor.

\section{Stability}

Stability is the degree of susceptibility to ambient disturbances in and around the biosensing system. These disturbances can cause a drift in the output signals of a biosensor under measurement. This can cause an error in the measured concentration and can affect the precision and accuracy of the biosensor. Stability is the most crucial feature in applications where a biosensor requires long incubation steps or continuous monitoring. The response of transducers and electronics can be temperature-sensitive, which may influence the stability of a biosensor. Therefore, appropriate tuning of electronics is required to ensure a stable response of the sensor. Another factor that can influence the stability is the affinity of the bioreceptor, which is the degree to which the analyte binds to the bioreceptor. Bioreceptors with high affinities encourage either strong electrostatic bonding or covalent linkage of the analyte that fortifies the stability of a biosensor. Another factor that affects the stability of a measurement is the degradation of the bioreceptor over a period of time.

\section{Sensitivity}

The minimum amount of analyte that can be detected by a biosensor defines its limit of detection (LOD) or sensitivity. In a number of medical and environmental monitoring applications, a biosensor is required to detect analyte concentration of as low as $\mathrm{ng} / \mathrm{ml}$ or even $\mathrm{fg} / \mathrm{ml}$ to confirm the presence of traces of analytes in a sample. For instance, a prostate-specific antigen (PSA) concentration of $4 \mathrm{ng} / \mathrm{ml}$ in blood is associated with prostate cancer for which doctors suggest biopsy tests. Hence, sensitivity is considered to be an important property of a biosensor.

\section{Linearity}

Linearity is the attribute that shows the accuracy of the measured response (for a set of measurements with different concentrations of analyte) to a straight line, mathematically represented as $y=m c$, where $c$ is the concentration of 
the analyte, $y$ is the output signal, and $m$ is the sensitivity of the biosensor. Linearity of the biosensor can be associated with the resolution of the biosensor and range of analyte concentrations under test. The resolution of the biosensor is defined as the smallest change in the concentration of an analyte that is required to bring a change in the response of the biosensor. Depending on the application, a good resolution is required as most biosensor applications require not only analyte detection but also measurement of concentrations of analyte over a wide working range. Another term associated with linearity is linear range, which is defined as the range of analyte concentrations for which the biosensor response changes linearly with the concentration.

\section{Applications of biosensors}

Biosensors have a very wide range of applications that aim to improve the quality of life. This range covers their use for environmental monitoring, disease detection, food safety, defence, drug discovery and many more. One of the main applications of biosensors is the detection of biomolecules that are either indicators of a disease or targets of a drug. For example, electrochemical biosensing techniques can be used as clinical tools to detect protein cancer biomarkers [14-16]. Biosensors can also be used as platforms for monitoring food traceability, quality, safety and nutritional value $[17,18]$. These applications fall into the category of 'single shot' analysis tools, i.e. where cost-effective and disposable sensing platforms are required for the application. On the other hand, an application such as pollution monitoring $[18,19]$ requires a biosensor to function from a few hours to several days. Such biosensors can be termed 'long-term monitoring' analysis tools. Whether it is long-term monitoring or single shot analysis, biosensors find their use as technologically advanced devices both in resource-limited settings and sophisticated medical set-ups: e.g. with applications in drug discovery [20-22]; for the detection of a number of chemical and biological agents that are considered to be toxic materials of defence interest [23]; for use in artificial implantable devices such as pacemakers [24] and other prosthetic devices [25]; and sewage epidemiology [26]. A range of electrochemical, optical and acoustic sensing techniques have been utilised, along with their integration into analytical devices for various applications. Figure 2 indicates different areas of research where biosensors have been used.

\section{Nanotechnology}

Irrespective of the field, miniaturisation has always proved to be beneficial for varied reasons. For instance, reducing the size of the biosensor to the micro- or nano-scale can result in a better signal-to-noise ratio as well as the possibility of using smaller sample volumes, which means lower assay costs. Moreover, when going towards nanoscale dimensions, the surface-to-volume ratio of the sensing active area increases and the sizes of the detecting electrode and that of the target biomarker become comparable. This causes both reduced non-specific binding and increased binding efficiency towards the target molecule. As a result, the bioreceptor becomes an active transducer for the sensing system and it becomes possible to perform single-molecule detection [27].

An interesting fact in an electrochemical system is that towards nanoscale dimensions the double layer capacitance dramatically decreases because of its dependence on the electrode area. As a result, the extremely low $R_{\mathrm{s}} C_{\mathrm{dl}}$ time constant (where $R_{\mathrm{s}}$ is the solution resistance, and $C_{\mathrm{dl}}$ is the double layer capacitance) allows ultra-fast electrontransfer kinetics and short-life intermediate species can also be investigated. As the time constant decreases, the time required to accomplish a measurement also diminishes towards the nanosecond domain. Moreover, when $C_{\mathrm{dl}} \mathrm{de}-$ creases dramatically, a further interesting consequence is the possibility of performing measurements in media with a high solution resistance where normal macroelectrodes are not usable. In fact, by keeping the $R_{\mathrm{s}} C_{\mathrm{dl}}$ factor constant, it is possible to perform measurements even without the need of a supporting electrolyte [28].

In terms of nanomaterials, the discovery of graphene and its oxidised form, graphene oxide, opened new frontiers in biosensors as well as in other research areas. Graphene is a pure form of carbon organised into single atom-thick sheets. This feature gives graphene exceptional chemical and physical properties.

The integration of graphene, graphene oxide and carbon nanotubes (single or multiple one atom-thick carbon concentric tubes) as well as nanoparticles and nanowires of different materials are widely reported in the literature for electrode fabrication. Biosensors so fabricated can nowadays allow limits of detection lower than previously possible, enabling even single-molecule detection. 


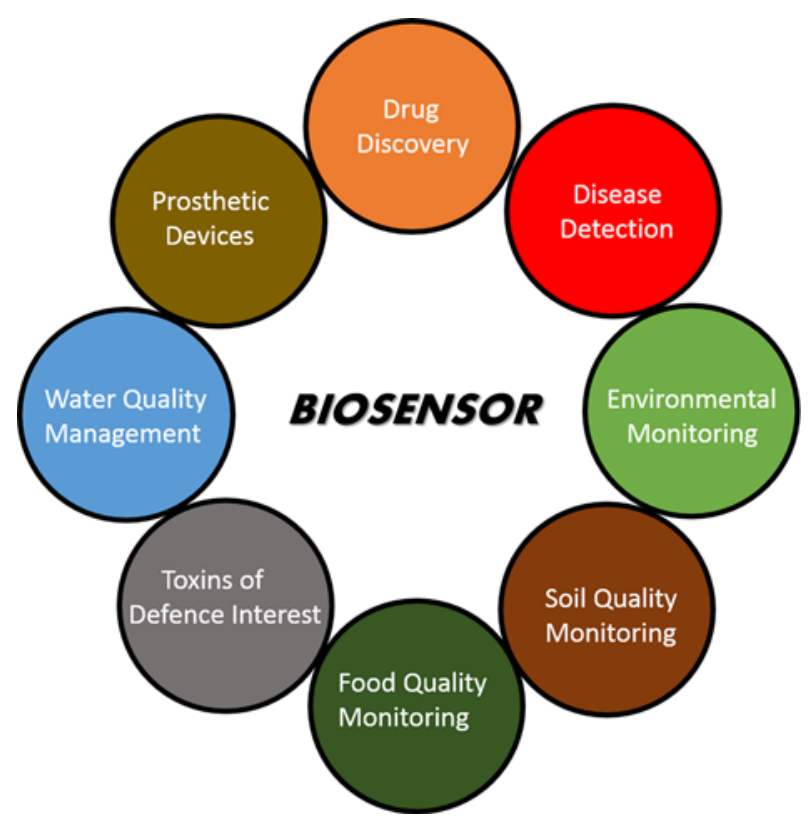

Figure 2. Major areas of applications for biosensors

\section{Impact of biosensors}

Looking at the never-ending literature related to biosensors over the last few decades, it undoubtedly reveals that biosensors are attractive not only in academia but also in industry. Biosensor technology exploits the unique properties of a biological recognition event on a transducing device. In such an event, the interaction of the analyte with the bioreceptor is converted into a suitable output that is easily readable by the user. This approach not only exploits the molecular binding event, but also brings researchers from different areas of science and engineering to bridge their skills. Similar practices have created an immense impact on early-stage researchers in the field of biosensors. In addition it has opened new frontiers in scientific research where considerable attention has been drawn towards the development of technologies to benefit different areas including healthcare. Working in an interdisciplinary field helps to think out of the box and work together with distinct professionals where every idea contributes to make something substantial. A simple example is a pregnancy test biosensor where researchers from biology highlighted the biological aspects and co-operated with engineers to work on the electronics of the system for the read-out. Finally, research from the laboratory is being transferred to customers worldwide because of management professionals. It would be naïve to think that biosensor research is confined to a niche - this can be seen clearly by the rapid increase in biosensors available in the market in recent times. Recently, there has been a gradual increase in start-up companies based on biosensor technology worldwide, which is having a profound impact on the healthcare industrial sector. In general, it can be said that biosensors have found an important place in our society as they aim to improve the quality of life in diverse areas such as homeland defence and security, agriculture, food safety, environment, medicine and pharmacology.

\section{Challenges in biosensing research}

Biosensors have been under development for around 50 years and the research in this field has made tremendous contributions in academia over the last 10 years. However, besides lateral flow pregnancy tests and electrochemical glucose biosensors, very few biosensors have achieved global commercial success at the retail level. There are several factors for this: difficulties in translating academic research into commercially viable prototypes by industry; complex regulatory issues in clinical applications; and it has not always been trivial to either find researchers with 
a background in biosensor technology or engage researchers from different disciplines of science and engineering to work together. Another reason is that academic research is driven by propositions of peer review of science, and funding agencies and politics that are sometimes characterised by various conflicts of interest. It is often a jury of academics who determine the priorities of funding agencies with legislators who seek considerable warrants for the funding they approve. If a subject can be made to appear fancy and attractive, it has a better chance of success. In this aspect, biosensor technology has a certain distinction that has been proficiently sold as a priority. Biosensors should be aimed as practical devices to be used. Although biosensors employ fundamental sciences, it can hardly be rationalised as 'curiosity-driven' research. On the other hand, research in industry obeys the trend of 'follow the money' to some extent. Given the success of commercial glucose sensors, biosensor research is, of course, very lucrative for the industry's long-term sustainability. However, it takes quite a long time to produce a commercially viable device from a proof of concept demonstrated in academia. This also involves a number of risks that industries are reluctant to face.

As a result there are unaddressed mandatory issues concerning the production of a commercial biosensor, such as:

- Identification of the market that is interested in a biosensor for a specific analyte of interest.

- Clear-cut advantages over existing methods for analyses of that analyte.

- Testing the performance of the biosensor both in use and after storage. Response of a biosensor after 6 months of storage is the absolute minimum for any practical commercial application.

- Stability, costs and ease of manufacturing each component of the biosensor.

- Hazards and ethics associated with the use of the developed biosensor.

The good news about biosensing technologies is that most of the barriers outlined above are being broken rapidly. High levels of investment have been poured into translational research worldwide, particularly, for healthcare applications. This is bringing industry closer to academia in order to provide commercially viable products. On the other hand, there has been an outstanding improvement in the way scientists work across boundaries. Engineering and physical scientists nowadays have a much better understanding of basic biomolecular processes, while biochemists and molecular biologists have greater awareness of the capabilities of different technologies. The alliance of experts of different disciplines from the onset of biosensing development projects is a very attractive proposition that will certainly bring advanced and novel products to the market.

\section{Conclusions}

In vitro molecular biosensors are nowadays ubiquitous in biomedical diagnosis as well as a wide range of other areas such as point-of-care monitoring of treatment and disease progression, environmental monitoring, food control, drug discovery, forensics and biomedical research. Biosensor devices require the interaction of different disciplines and rely on very distinct aspects such as the study of interactions of bio-recognition elements with biomolecular analytes, immobilisation of biomolecules on to solid surfaces, development of anti-fouling surface chemistries, device design and fabrication, integration of biology with the devices, microfluidics, on-chip electronics, packaging, sampling techniques, etc.

The rapid development in the field of biosensors over the past decades, both at the research and product development level, is due mainly to: (i) developments in miniaturisation and microfabrication technologies; (ii) the use of novel bio-recognition molecules; (iii) novel nanomaterials and nanostructured devices; and (iv) better interaction between life scientists and engineering/physical scientists.

A range of target molecules and affinity reagents can be used for a wide range of biosensors. Antibody-based systems (Chapter 2) represent the gold standard in biosensors. Novel affinity reagents such as synthetic receptors are currently making way to replace antibodies on biosensors, in particular, aptamers such as peptide aptamers (Chapter 3) and oligonucleotide aptamers (Chapter 4). DNA and oligonucleotide analogues such as peptide nucleic acids (PNAs) and locked nucleic acids (LNAs) are often used as probe molecules for DNA and microRNA (miRNA) sensing (Chapter 4). Determination of protein glycosylation levels using lectins is currently of great interest in medical diagnosis (Chapter 5) as is the sensing of toxins in environmental monitoring (Chapter 6). Suitable bioconjugation strategies and stabilisation of biomolecules on electrodes is essential for the development of commercially viable biosensors (Chapter 7).

A range of transduction techniques can be used in biosensing devices, including electrochemical sensors (Chapter 8), field-effect transistors (Chapter 9), optical sensors (Chapter 10) and acoustic-sensitive sensors (Chapter 11). 
Lateral flow systems (Chapter 12) have great promise for the development of inexpensive and easy to use point-ofcare sensors beyond the traditional pregnancy tests, whereas lab-on-chip devices (Chapter 13) integrate different microfabrication techniques enabling biosensors to be employed in a wide range of applications using minute sample volumes and minimum sample preparation.

\section{Summary}

- Biosensors are nowadays ubiquitous in different areas of healthcare.

- Pregnancy tests and glucose monitoring sensors are the two main examples of very successful biosensor devices.

- A range of transduction techniques such as electrochemical, optical and acoustic, can be used for biosensors.

- High-affinity reagents such as antibodies, enzymes and synthetic biomolecules can be coupled to the transducer in order to provide specificity of the biosensors.

- Nanotechnology has had a major impact on recent advances of biosensing technology.

\section{Funding}

We acknowledge funding from the European Commission Framework Programme through the Marie Curie Initial Training Network PROSENSE [grant number 317420, 2012-2016].

\section{Competing Interests}

The Authors declare that there are no competing interests associated with the manuscript.

\section{References}

1 Cremer, M. (1906) Über die Ursache der elektromotorischen Eigenschaften der Gewebe, zugleich ein Beitrag zur Lehre von den polyphasischen Elektrolytketten. Z. Biol. 47, 562-608

2 Hughes, W.S. (1922) The potential difference between glass and electrolytes in contact with the glass. J. Am. Chem. Soc. 44, 2860-2867 CrossRef

3 Griffin, E.G. and Nelson, J.M. (1916) The influence of certain substances on the activity of invertase. J. Am. Chem. Soc. 38, 722-730 CrossRef

4 Nelson, J.M. and Griffin, E.G. (1916) Adsorption of invertase. J. Am. Chem. Soc. 38, 1109-1115 CrossRef

5 Heineman, W.R. and Jensen, W.B. (2006) Leland C. Clark Jr. (1918-2005). Biosens. Bioelectron. 21, 1403-1404 CrossRef

6 Guilbault, G.G. and Montalvo, Jr, J.G. (1969) Urea-specific enzyme electrode. J. Am. Chem. Soc. 91, 2164-2165 CrossRef PubMed

7 Bergveld, P. (1970) Development of an ion-sensitive solid-state device for neurophysiological measurements. IEEE Trans. Biomed. Eng. 1 (7), 70-71 CrossRef

8 Vestergaard, M.C., Kerman, K., Hsing, I.M. and Tamiya, E. (eds). (2015) Nanobiosensors and Nanobioanalyses, Springer, Tokyo

9 Yoo, E.H. and Lee, S.Y. (2010) Glucose biosensors: an overview of use in clinical practice. Sensors 10, 4558-4576 CrossRef PubMed

10 Suzuki, S., Takahashi, F., Satoh, I. and Sonobe, N. (1975) Ethanol and lactic acid sensors using electrodes coated with dehydrogenase-collagen membranes. Bull. Chem. Soc. Jpn. 48, 3246-3249 CrossRef

11 Schultz, J.S. (1982) Optical sensor of plasma constituents. U.S. Pat. 4,344,438 A

12 Liedberg, B., Nylander, C. and Lunström, I. (1983) Surface plasmon resonance for gas detection and biosensing. Sens. Actuators 4, 299-304 CrossRef

13 Cass, A.E., Davis, G., Francis, G.D., Hill, H.A.O., Aston, W.J., Higgins, I.J. et al. (1984) Ferrocene-mediated enzyme electrode for amperometric determination of glucose. Anal. Chem. 56, 667-671 CrossRef PubMed

14 Jolly, P., Formisano, N. and Estrela, P. (2015) DNA aptamer-based detection of prostate cancer. Chem. Pap. 69, 77-89 CrossRef

15 Jolly, P., Formisano, N., Tkáć, J., Kasák, P., Frost, C.G. and Estrela, P. (2015) Label-free impedimetric aptasensor with antifouling surface chemistry: a prostate specific antigen case study. Sens. Actuators B 209, 306-312 CrossRef

16 Formisano, N., Jolly, P., Bhalla, N., Cromhout, M., Flanagan, S.P. and Fogel, R. et al. (2015) Optimisation of an electrochemical impedance spectroscopy aptasensor by exploiting quartz crystal microbalance with dissipation signals. Sens. Actuators B 220, 369-375 CrossRef

17 Sharma, T.K., Ramanathan, R., Rakwal, R., Agrawal, G.K. and Bansal, V. (2015) Moving forward in plant food safety and security through nanobiosensors: adopt or adapt biomedical technologies? Proteomics 15, 1680-1692 CrossRef PubMed

18 Van Dorst, B., Mehta, J., Bekaert, K., Rouah-Martin, E., De Coen, W., Dubruel, P. et al. (2010) Recent advances in recognition elements of food and environmental biosensors: a review. Biosens. Bioelectron. 26, 1178-1194 CrossRef PubMed 
19 Gavrilescu, M., Demnerová, K., Aamand, J., Agathos, S. and Fava, F. (2015) Emerging pollutants in the environment: present and future challenges in biomonitoring, ecological risks and bioremediation. N. Biotechnol. 32, 147-156 CrossRef

20 Bhalla, N., Di Lorenzo, M., Pula, G. and Estrela, P. (2014) Protein phosphorylation analysis based on proton release detection: potential tools for drug discovery. Biosens. Bioelectron. 54, 109-114 CrossRef PubMed

21 Bhalla, N., Di Lorenzo, M., Pula, G. and Estrela, P. (2015) Protein phosphorylation detection using dual-mode field-effect devices and nanoplasmonic sensors. Sci. Rep. 5, 8687 CrossRef PubMed

22 Bhalla, N., Formisano, N., Miodek, A., Jain, A., Di Lorenzo, M., Pula, G. et al. (2015) Plasmonic ruler on field-effect devices for kinase drug discovery applications. Biosens. Bioelectron. 71, 121-128 CrossRef PubMed

23 Paddle, B.M. (1996) Biosensors for chemical and biological agents of defence interest. Biosens. Bioelectron. 11, 1079-1113 CrossRef PubMed

24 Grayson, A.C.R., Shawgo, R.S., Johnson, A.M., Flynn, N.T., Li, Y., Cima, M.J. et al. (2004) A BioMEMS review: MEMS technology for physiologically integrated devices. Proc. IEEE 92, 6-21 CrossRef

25 Parker, K.K. and 0'Grady, M. (2015) Porous electroactive hydrogels and uses thereof. U.S. Pat. 8,999,378 B2.

26 Yang, Z., Kasprzyk-Hordern, B., Frost, C.G., Estrela, P. and Thomas, K.V. (2015) Community sewage sensors for monitoring public health. Environ. Sci. Technol. 49, 5845-5846 CrossRef PubMed

27 Adams, K.L., Puchades, M. and Ewing, A.G. (2008) In vitro electrochemistry of biological systems. Annu. Rev. Anal. Chem. (Palo Alto Calif.) 1, 329-355 CrossRef

28 Zhang, Y., Zhang, B. and White, H.S. (2006) Electrochemistry of nanopore electrodes in low ionic strength solutions. J. Phys. Chem. B 110, 1768-1774 CrossRef PubMed 